\title{
Effect of three common IL-17 single nucleotide polymorphisms on the risk of developing gastric cancer
}

\author{
YA-WEN GAO ${ }^{1}$, MEILI XU ${ }^{1}$, YAN XU $^{1}$, DAN LI $^{1}$ and SHENGHUA ZHOU ${ }^{2}$ \\ Departments of ${ }^{1}$ Gerontology and ${ }^{2}$ Vasculocardiology, The Second Xiangya Hospital, Central South University, \\ Changsha, Hunan 410011, P.R. China
}

Received March 20, 2014; Accepted October 24, 2014

DOI: 10.3892/ol.2014.2827

\begin{abstract}
A 1:1 matched case-control study was conducted to analyze the association between three common interleukin (IL)-17A and IL-17F single nucleotide polymorphisms (SNPs) and the risk of developing gastric cancer. Genotyping of SNPs rs2275913, rs763780 and rs3748067 within the IL-17 gene were detected by performing polymerase chain reaction-restriction fragment length polymorphism analysis. Gastric cancer patients were more likely to be cigarette smokers, alcohol drinkers and have a family history of cancer in their first-degree relatives. Patients carrying the rs763780 polymorphism were correlated with a significant increased risk of gastric cancer in codominant, dominant and recessive models. Additionally, individuals with the rs763780 polymorphism were correlated with a markedly increased risk of gastric cancer among alcohol drinkers in codominant, dominant and recessive models. Furthermore, a significant correlation was identified between the rs 763780 polymorphism and the consumption of alcohol. However, no association was identified between rs2275913 and rs3748067 polymorphisms and the risk of developing gastric cancer. Thus, the present study reported that the rs763780 polymorphism may be associated with risk of developing gastric cancer in the population studied, particularly in alcohol drinkers.
\end{abstract}

\section{Introduction}

Gastric cancer is one of the most common types of cancer and is associated with a high incidence of mortality worldwide $(1,2)$. Although genetic and environmental factors in addition to Helicobacter pylori infections have been identified to be important in the development of gastric cancer (3-5), the precise etiology of the disease remains unclear. Numerous studies have reported that inflammation-associated gene

Correspondence to: Mr. Shenghua Zhou, Department of Vasculocardiology, The Second Xiangya Hospital, Central South University, 139 Renmin Road, Changsha, Hunan 410011, P.R. China E-mail: zhoushenghua_csu@163.com

Key words: IL-17, gastric cancer, polymorphism polymorphisms may be involved in the development of gastric cancer, including tumor necrosis factor $\alpha$ and interleukin (IL) genes (6-8).

IL-17 is a cytokine that is secreted exclusively by activated $\mathrm{T}$ cells, which bridge the adaptive and innate immune systems $(9,10)$. IL-17A and IL-17F are important members of the IL-17 cytokine family; they are preferentially produced by T helper 17 (Th17) cells, which are responsible for the pathogenic activity of the lineage of cluster of differentiation (CD) $4^{+}$ effector cells and multiple proinflammatory mediators $(11,12)$. A previous study reported that IL-17A and IL-17F single nucleotide polymorphisms (SNPs) are associated with the risk of developing gastric cancer (13). However, subsequent replication studies investigating the association between IL-17A and IL-17F variants with the risk of developing gastric cancer were controversial $(10,14-16)$. This discrepancy may be attributed to the relatively small sample size of previous studies and the genetic heterogeneity of polymorphisms in gastric cancer among different populations.

Therefore, to clarify the conflicting findings of previous reports, the present study used multiple genetic statistical models to conduct a 1:1 matched case-control study to analyze the association between three common IL-17A and IL-17F SNPs and the risk of developing gastric cancer in the study population.

\section{Materials and methods}

Study population. Between May 2010 and May 2012,572 gastric cancer patients were recruited from the Second Xiangya Hospital of Central South University (Changsha, China); the gastric cancer patients were recently and histopathologically diagnosed with primary gastric cancer. The exclusion criteria were as follows: Patients who exhibited secondary or recurrent tumors, a history of other malignant neoplasm, or inadequate organ function. Gender and age-matched $( \pm 5$ years $)$ individuals were selected from those who visited the Second Xiangya Hospital for a routine health check-up. Additionally, 572 controls were selected from inpatients in the Departments of Orthopedics and Dermatology, and were matched to the selected gastric cancer patients by age ( \pm 5 years) and gender. None of the control patients had a history of cancer. Written informed consent was obtained from all of the patients prior to participation in the present study and the protocol of the 
present study was approved by the ethics committee of the Second Xiangya Hospital of Central South University.

A self-designed questionnaire was developed to investigate the demographic characteristics of all case and control patients. All of the patients completed the questionnaire, which was conducted by trained interviewers who were not aware of the study hypothesis. H. pylori infection status was evaluated using histological examination or a rapid urea breath test; if one of the tests indicated a positive result, the patients was diagnosed as positive for $H$. pylori infection.

Genotype analysis. All of the study participants provided a $5-\mathrm{ml}$ venous blood sample, which was stored at $-20^{\circ} \mathrm{C}$ until required. According to the manufacturer's instructions, genomic DNA was extracted from the peripheral venous blood samples using the TIANamp blood DNA kit (Tiangen Biotech Co., Ltd., Beijing, China). Genotyping of SNPs rs2275913,rs3748067 and rs763780 within the IL-17 gene were detected by performing polymerase chain reaction-restriction fragment length of polymorphism (PCR-RFLP) analysis. The primers used for SNPs rs2275913, rs3748067 and rs763780 were designed using MassARRAY ${ }^{\circledR}$ Assay Design software version 3.1 (Sequenom, San Diego, CA, USA) according to the manufacturer's instructions (Table I). The cycling program involved preliminary denaturation at $95^{\circ} \mathrm{C}$ for $5 \mathrm{~min}$, followed by 35 cycles of denaturation at $95^{\circ} \mathrm{C}$ for $30 \mathrm{sec}$, annealing at $62^{\circ} \mathrm{C}$ for $30 \mathrm{sec}, 72^{\circ} \mathrm{C}$ for $30 \mathrm{sec}$ and a final extension at $72^{\circ} \mathrm{C}$ for $10 \mathrm{~min}$. The digested PCR products were run on a $2 \%$ agarose gel stained with ethidium bromide and ultraviolet light, followed by sequencing of the PCR products using an automated sequencing system (PTC-200 DNA Engine PThermal Cycler; MJ Research, Inc., Waltham, MA, USA).

Statistical analysis. Continuous variables are presented as the mean \pm standard deviation and were analyzed using the independent sample Student's t-test. Categorical variables are presented as frequencies (percentages) and were analyzed using the $\chi^{2}$ test. The Hardy-Weinberg equilibriums between groups were compared using the $\chi^{2}$ test. A conditional multiple logistical regression model was used to assess the effects of SNPs rs2275913, rs3748067 and rs763780 on gastric cancer risk; the results were adjusted for potential confounding variables prior to their expression as odds ratios (ORs) and 95\% confidence intervals (CIs). The homozygous genotypes of the three SNPs were used as the reference group. All $\mathrm{P}$-values were two sided, and $\mathrm{P}<0.05$ was considered to indicate a statistically significant difference. All statistical analyses were performed using SPSS software, version 11.0 (SPSS, Inc., Chicago, IL, USA) for Windows.

\section{Results}

Characteristics of patients and controls. The characteristics of the case and control patients are demonstrated in Table II. As expected, no significant difference was identified between the case and control patients in terms of gender or age $(\mathrm{P}>0.05)$. The gastric cancer cases were more likely to be cigarette smokers, alcohol drinkers and have a history of cancer in their first-degree relatives. However, no significant difference was identified in the gender, age, alcohol consumption or annual income between the case and control patients.

Multivariate analysis. The genotype distributions of IL-17 rs2275913 and rs763780 among the controls were within the parameters of the Hardy-Weinberg equilibrium; however, the distributions of IL-17 rs3748067 were not. Using conditional regression analysis, it was identified that individuals carrying the IL-17 rs763780 CC genotype demonstrated a marginally increased risk of developing gastric cancer when compared with the TT genotype in the codominant model, with an adjusted OR (95\% CI) of 2.27 (1.51-3.45) (Table III). Furthermore, the present study identified that individuals carrying the rs763780 polymorphism were correlated with a significantly increased risk of developing gastric cancer in dominant and recessive models, demonstrating an OR $(95 \% \mathrm{CI})$ of 1.71 (1.27-2.30) and 2.20 (1.47-3.34), respectively. However, no significant association was observed between rs2275913 and rs3748067 polymorphisms, and the risk of developing gastric cancer.

Association between IL-17 genotypes and environmental factors. Additionally, the present study investigated the interaction between the rs763780 polymorphism with the risk of developing gastric cancer via cigarette smoking, alcohol consumption and $H$. pylori infection (Table IV). Individuals with the rs763780 TT genotype who were alcohol drinkers were significantly associated with an increased risk of developing gastric cancer (OR,4.27; 95\% CI, 2.24-8.63). Furthermore, the rs763780 polymorphism was correlated with an increased risk of developing gastric cancer among alcohol drinkers in dominant and recessive models [OR (95\% CI), 2.34 (1.47-3.78); OR (95\%CI), 4.19 (2.20-8.41), respectively]. Thus, a significant interaction was identified between the rs763780 polymorphism and alcohol consumption in gastric cancer risk $(\mathrm{P}=0.02)$. However, no significant interaction was identified between the rs763780 polymorphism and cigarette smoking or $H$. pylori infection in the risk of developing gastric cancer.

\section{Discussion}

Identification of genes involved in the genetic predisposition to or progression of cancer is important in clinical practice and in basic medical research. IL-17A and IL-17F are expressed by Th17 cells and are involved in coordinating local tissue inflammation $(17,18)$. Various studies have demonstrated that IL-17A and IL-17F may be involved in the development of gastric cancer $(15,16,19)$, however, the results were unclear. Therefore, the present study conducted a case-control investigation to provide a more reliable conclusion of the association between the IL-17A and IL-17F SNPs, and gastric cancer.

The rs 2275913 polymorphism is located at the 5 ' region of the IL-17A gene and, therefore, may regulate gene transcription (20). By contrast, the rs763780 polymorphism is located in the coding region and is a missense mutation, which may influence the protein structure and function $(19,21)$. Hence, the functions of these two SNPs requires further investigation.

IL-17 family members are a cytokines and are involved in coordinating local tissue inflammation by the release of proinflammatory and neutrophil-mobilizing cytokines (21). 
Table I. Primers and PCR-RFLP analysis for VEGF genetic polymorphisms.

\begin{tabular}{llr}
\hline SNP & \multicolumn{1}{c}{ Primer sequence } & Amplification fragment, bp \\
\hline rs2275913 & Forward, 5'-GCCCTTCCCATTTTCCTTCAGA-3' & 210 \\
rs763780 & Reverse, 5'-CCAATCAACTGGGGATGGATGA-3' & 188 \\
& Forward, 5'-CTGTTTCCATCCGTGCAGGTC-3' & \\
rs3748067 & Reverse, 5'-TGGTGACTGTTGGCTGCACCT-3' & 217 \\
& Forward, 5'-AAGCAGGGAGCCTGCAGAGTG-3' \\
& Reverse, 5'-GGCACCACACAACCCAGAAAG-3' & \\
\hline
\end{tabular}

PCR-RFLP, polymerase chain reaction-restriction fragment length polymorphism; VEGF, vascular endothelial growth factor; SNP, single nucleotide polymorphism.

Table II. Demographic characteristics of included cases and controls.

\begin{tabular}{|c|c|c|c|c|c|c|}
\hline \multirow[b]{2}{*}{ Variable } & \multicolumn{2}{|c|}{ Cases $(n=572)$} & \multicolumn{2}{|c|}{ Controls $(n=572)$} & \multirow[b]{2}{*}{$t$ or $\chi^{2}$} & \multirow[b]{2}{*}{ P-value } \\
\hline & $\mathrm{n}$ & $\%$ & $\mathrm{n}$ & $\%$ & & \\
\hline \multicolumn{7}{|l|}{ Age, years } \\
\hline$<55$ & 249 & 43.53 & 253 & 44.23 & & \\
\hline$\geq 55$ & 323 & 56.47 & 319 & 55.77 & 0.06 & 0.810 \\
\hline \multicolumn{7}{|l|}{ Gender } \\
\hline Female & 220 & 38.46 & 220 & 38.46 & & \\
\hline Male & 352 & 61.54 & 352 & 61.54 & 0.00 & 1.000 \\
\hline \multicolumn{7}{|c|}{ Cancer history in first-degree relatives } \\
\hline No & 522 & 91.26 & 569 & 99.48 & & \\
\hline Yes & 50 & 8.74 & 3 & 0.52 & 43.70 & $<0.001$ \\
\hline \multicolumn{7}{|c|}{ Alcohol drinker } \\
\hline Never & 333 & 58.22 & 375 & 65.56 & & \\
\hline Ever & 239 & 41.78 & 197 & 34.44 & 6.54 & 0.010 \\
\hline \multicolumn{7}{|c|}{ Cigarette smoker } \\
\hline Never & 386 & 67.48 & 424 & 74.13 & & \\
\hline Ever & 186 & 32.52 & 148 & 25.87 & 6.11 & 0.010 \\
\hline \multicolumn{7}{|c|}{ Helicobacter pylori } \\
\hline Negative & 201 & 35.14 & 308 & 53.85 & & \\
\hline Positive & 371 & 64.86 & 266 & 46.50 & 39.80 & $<0.001$ \\
\hline
\end{tabular}

Table III. Genotype frequencies of three SNPs of IL-17A and IL-17F in gastric cancer patients and controls.

\begin{tabular}{|c|c|c|c|c|c|c|c|c|}
\hline \multirow[b]{2}{*}{ Genotype } & \multicolumn{2}{|c|}{ Cases $(n=572)$} & \multicolumn{2}{|c|}{ Controls $(\mathrm{n}=572)$} & \multirow[b]{2}{*}{$\begin{array}{c}\text { Hardy-Weinberg } \\
\text { equilibrium }\end{array}$} & \multicolumn{3}{|c|}{ OR $(95 \% \mathrm{CI})$} \\
\hline & $\mathrm{n}$ & $\%$ & $\mathrm{n}$ & $\%$ & & $\begin{array}{l}\text { Codominant } \\
\text { model }\end{array}$ & $\begin{array}{l}\text { Dominant } \\
\text { model }\end{array}$ & $\begin{array}{l}\text { Recessive } \\
\text { model }\end{array}$ \\
\hline \multicolumn{9}{|l|}{ rs2275913 } \\
\hline GG & 239 & 41.8 & 260 & 45.4 & & Ref. (1.0) & & \\
\hline GA & 250 & 43.7 & 241 & 42.1 & & $1.13(0.87-1.46)$ & & \\
\hline AA & 83 & 14.5 & 72 & 12.5 & 0.17 & $1.25(0.86-1.83)$ & $1.16(0.91-1.47)$ & $1.18(0.83-1.68)$ \\
\hline \multicolumn{9}{|l|}{ rs763780 } \\
\hline $\mathrm{CC}$ & 420 & 73.5 & 472 & 82.5 & & Ref. (1.0) & & \\
\hline $\mathrm{CT}$ & 67 & 11.7 & 58 & 10.2 & & $1.30(0.88-1.93)$ & & \\
\hline $\mathrm{TT}$ & 85 & 14.8 & 42 & 7.3 & 0.09 & $2.27(1.51-3.45)$ & $1.71(1.27-2.30)$ & $2.20(1.47-3.34)$ \\
\hline \multicolumn{9}{|l|}{ rs3748067 } \\
\hline $\mathrm{TT}$ & 460 & 80.4 & 458 & 80.4 & & Ref. (1.0) & & \\
\hline $\mathrm{TC}$ & 70 & 12.2 & 66 & 11.5 & & $1.06(0.72-1.54)$ & & \\
\hline $\mathrm{CC}$ & 42 & 7.4 & 47 & 8.1 & $<0.001$ & $0.89(0.56-1.41)$ & $0.99(0.90-1.69)$ & $0.90(0.57-1.42)$ \\
\hline
\end{tabular}

SNP, single nucleotide polymorphism; IL, interleukin; OR, odds ratio; CI, confidence interval; Ref. the GG genotype is the reference for the GA genotype. 
Polymorphisms in IL-17 cytokines alter the activity of interleukins and may alter cytokine function, thus, dysregulating IL-17 expression (12). Previous studies reported that IL-17 polymorphisms are associated with the risk of developing gastric cancer in Japanese, Chinese and Iranian populations, however, these genetic polymorphisms appear to exhibit different effects in each populations $(10,13-16,19)$. Shibata et al (10) reported that IL-17 rs2275913 polymorphism was significantly associated with the development of gastric cancer; however, Wu et al (13) reported that while the rs763780 polymorphism was involved in the development of gastric cancer, the rs2275913 polymorphism was not. In the present study, it was identified that the rs763780 polymorphism appears to be associated with the risk of developing gastric cancer, as well as interacting with the variable of alcohol consumption. The discrepancy of the results of the present study and previous studies may be explained by differences in the ethnicities of the participants, the source of the control subjects, the sample size, or by chance. Additional studies are required to clarify the association between IL-17 polymorphisms and the risk of developing gastric cancer.

The present study was limited by a number of factors. First, the study was conducted in a single hospital in Changsha, China; therefore, the participants may not have been representative of the Chinese population as a whole. Second, the etiology of gastric cancer involves multiple genes and environmental factors, which were not considered in the present study. Third, the genotypic distributions of rs3748067 in the case and control patients were not consistent with Hardy-Weinberg equilibriums, which indicates that the cohort of the present study may be a poor representation of the Chinese population. Finally, the predominant limitation of the present study was the relatively small sample size, therefore, additional large sample size studies are required to confirm our results.

In conclusion, the present study reported that the rs763780 polymorphisms may be associated with risk of developing gastric cancer in a Chinese population, particularly in alcohol drinkers. However, no association was identified between the rs2275913 and rs3748067 polymorphisms and the risk of gastric cancer. Therefore, the rs763780 polymorphism may be used as a diagnostic biomarker for gastric cancer. Additional large sample size studies are required to confirm the role of IL-17 polymorphisms in the development of gastric cancer.

\section{References}

1. Hartgrink HH, Jansen EP, van Grieken NC and van de Velde CJ: Gastric cancer. Lancet 374: 477-490, 2009.

2. Siegel R, Naishadham D and Jemal A: Cancer statistics, 2012. CA Cancer J Clin 62: 10-29, 2012.

3. Houghton J and Wang TC: Helicobacter pylori and gastric cancer: a new paradigm for inflammation-associated epithelial cancers. Gastroenterology 128: 1567-1578, 2005.

4. Merchant JL: Inflammation, atrophy, gastric cancer: connecting the molecular dots. Gastroenterology 129: 1079-1082, 2005.

5. Dong LM, Potter JD, White E, et al: Genetic susceptibility to cancer: the role of polymorphisms in candidate genes. JAMA 299: 2423-2436, 2008.

6. El-Omar EM, Carrington M, Chow WH, et al: Interleukin-1 polymorphisms associated with increased risk of gastric cancer. Nature 404: 398-402, 2000.

7. Xue $\mathrm{H}$,Lin B, An J, et al: Interleukin-10-819 promoter polymorphism in association with gastric cancer risk. BMC Cancer 12: 102, 2012. 
8. Zhu F, Zhao H, Tian X and Meng X: Association between tumor necrosis factor- $\alpha$ rs1800629 polymorphism and risk of gastric cancer: a meta-analysis. Tumour Biol 35: 1799-1803, 2014.

9. Moseley TA, Haudenschild DR, Rose L and Reddi AH: Interleukin-17 family and IL-17 receptors. Cytokine Growth Factor Rev 14: 155-174, 2003

10. Shibata T, Tahara T, Hirata I and Arisawa T: Genetic polymorphism of interleukin-17A and -17F genes in gastric carcinogenesis. Hum Immunol 70: 547-551, 2009.

11. Rutitzky LI, Lopes da Rosa JR and Stadecker MJ: Severe CD4 $\mathrm{T}$ cell-mediated immunopathology in murine schistosomiasis is dependent on IL-12p40 and correlates with high levels of IL-17. J Immunol 175: 3920-3926, 2005.

12. Ishigame $\mathrm{H}$, Kakuta $\mathrm{S}$, Nagai $\mathrm{T}$, et al: Differential roles of interleukin-17A and $-17 \mathrm{~F}$ in host defense against mucoepithelial bacterial infection and allergic responses. Immunity 30: 108-119, 2009.

13. Wu X, Zeng Z, Chen B, et al: Association between polymorphisms in interleukin-17A and interleukin-17F genes and risks of gastric cancer. Int J Cancer 127: 86-92, 2010.

14. Arisawa T, Tahara T, Shiroeda $\mathrm{H}$, et al: Genetic polymorphisms of IL17A and pri-microRNA-938, targeting IL17A 3'-UTR, influence susceptibility to gastric cancer. Hum Immunol 73: 747-752, 2012

15. Rafiei A, Hosseini V, Janbabai G, et al: Polymorphism in the interleukin-17A promoter contributes to gastric cancer. World J Gastroenterol 19: 5693-5699, 2013.
16. Zhang X, Zheng L, Sun Y and Zhang X: Analysis of the association of interleukin-17 gene polymorphisms with gastric cancer risk and interaction with Helicobacter pylori infection in a Chinese population. Tumour Biol 2014: 1575-1580, 2014.

17. Lee DY, Hong SW, Chang YG, Lee WY and Lee B: Clinical significance of preoperative inflammatory parameters in gastric cancer patients. J Gastric Cancer 13: 111-116, 2013.

18. Park H, Li Z, Yang XO, et al: A distinct lineage of CD4 T cells regulates tissue inflammation by producing interleukin 17 . Nat Immunol 6: 1133-1141, 2005.

19. Qinghai Z, Yanying W, Yunfang C, Xukui Z and Xiaoqiao Z: Effect of interleukin-17A and interleukin-17F gene polymorphisms on the risk of gastric cancer in a Chinese population. Gene 537: 328-332, 2014

20. Arisawa T, Tahara T, Shibata T, et al: The influence of polymorphisms of interleukin-17A and interleukin-17F genes on the susceptibility to ulcerative colitis. J Clin Immunol 28: 44-49, 2008

21. Kawaguchi M1, Adachi M, Oda N, Kokubu F and Huang SK: IL-17 cytokine family. J Allergy Clin Immunol 114: 1265-1273, 2004. 